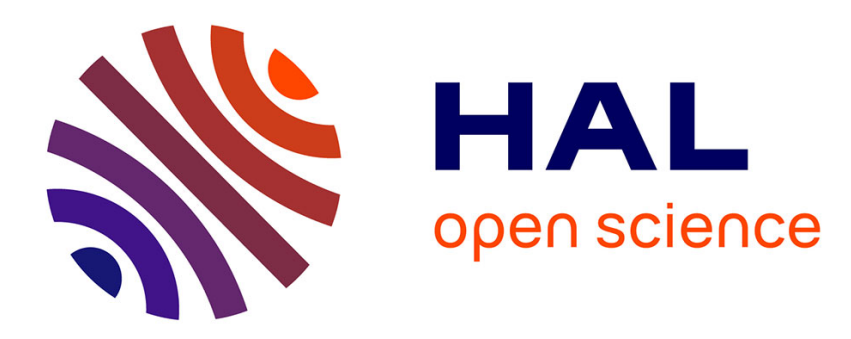

\title{
Variance Estimation in the Central Limit Theorem for Markov chains
}

\author{
Samis Trevezas, Nikolaos Limnios
}

\section{To cite this version:}

Samis Trevezas, Nikolaos Limnios. Variance Estimation in the Central Limit Theorem for Markov chains. Journal of Statistical Planning and Inference, 2009, 139 (7), pp.2242-2253. 10.1016/j.jspi.2008.10.020 . inria-00468804

\section{HAL Id: inria-00468804 https://hal.inria.fr/inria-00468804}

Submitted on 31 Mar 2010

HAL is a multi-disciplinary open access archive for the deposit and dissemination of scientific research documents, whether they are published or not. The documents may come from teaching and research institutions in France or abroad, or from public or private research centers.
L'archive ouverte pluridisciplinaire HAL, est destinée au dépôt et à la diffusion de documents scientifiques de niveau recherche, publiés ou non, émanant des établissements d'enseignement et de recherche français ou étrangers, des laboratoires publics ou privés. 


\title{
Variance Estimation in the Central Limit Theorem for Markov Chains
}

\author{
Samis TrevezAs* Nikolaos Limnios \\ Laboratoire de Mathématiques Appliquées de Compiègne \\ Université de Technologie de Compiègne
}

\begin{abstract}
This article concerns the variance estimation in the central limit theorem for finite recurrent Markov chains. The associated variance is calculated in terms of the transition matrix of the Markov chain. We prove the equivalence of different matrix forms representing this variance. The maximum likelihood estimator for this variance is constructed and it is proved that it is strongly consistent and asymptotically normal. The main part of our analysis consists in presenting closed matrix forms for this new variance. Additionally, we prove the asymptotic equivalence between the empirical and the MLE estimator for the stationary distribution.
\end{abstract}

Key words: Markov Chain estimation, Central limit theorem, Variance estimation

AMS Subject Classification: 62M05, 60F05, 60J10, 62F12

* Corresponding author
Email address: streveza@utc.fr (Samis TREvEzAs). 


\section{Introduction and Preliminaries}

Markov processes (MP) are a central topic in applied probability and statistics. The reason is that many real problems can be modeled by this kind of stochastic processes in continuous or in discrete time. Statistical estimation for finite state ergodic Markov chains (MC) was discussed by Billingsley (1961b), Billingsley (1961a). Estimation consists in considering a trajectory observed on a time interval $\{0,1, \ldots, m\}$ and then give an estimate for the transition function. Other quantities can also be estimated, as the stationary distribution, the mean hitting times and relevant probabilistic characteristics which are involved in the analysis of a MC. To this purpose, an important problem is to estimate the variance $\sigma^{2}$ which appears in CLTs. In particular, when we consider additive functionals of a MC, this variance is the same as in the functional central limit theorems (FCLT) for MC. Recent papers concentrate on this aspect for MCMC settings. A recent survey is due to Roberts and Rosenthal (2004). Chauveau and Diebolt (2003) study a continuous-time empirical variance process based on i.i.d. parallel chains. Stefanov (1995) considers also maximum likelihood estimation (MLE) for MP and MC via the exponential families approach and derives closed form solutions for the variance parameters in FCLTs. The CLT is still an aspect of the MC which worth being studied even in a countable or in a finite state space. To this end our work focus the attention on computational aspects for the MLE of the associated variance. This paper is organized as follows. In Section 2 we study and present the associated variance on the CLT in different matrix forms. These different forms result from different proofs of the CLT found already in the literature with some slight variations in the terminology. For some different proofs see Doob (1953), Billingsley (1961b), Dacunha-Castelle and Duflo (1983), Meyn and Tweedy (1993), Port (1994). The starting point for our analysis is the 
form for $\sigma^{2}$ given in Port (1994). In the first part of Section 3 we review some basic facts on the estimation of the stationary distribution and we prove the equivalence between the MLE and the empirical estimator of the stationary distribution. Our main task is accomplished in the second part of Section 3, where the MLE of the variance is constructed in one of its matrix forms and proved to be strongly consistent and asymptotically normal with asymptotic variance computed in two different matrix forms. Finally, we present some examples in order to enlighten the theoretical results.

Let $\mathbf{X}=\left(X_{n} ; n \in \mathbb{N}\right)$ be a Markov chain which we assume (except for Theorem 1 which is more generally valid on countable state space) to have finite state space $E=\{1,2, \ldots, s\}$ and be defined on a probability space $(\Omega, \mathscr{F}, \mathbb{P})$. The values belonging to $E$ will represent here the states of an observed system. It is assumed that $X$ is irreducible with transition matrix $P$ and stationary distribution $\boldsymbol{\pi}$. We are going to use the following notation.

- $f: E \rightarrow \mathbb{R} ; f$ will be understood here as a reward function associated with the states of the system.

- $S_{n}=\sum_{i=0}^{n} f\left(X_{i}\right) ; S_{n}$ stands for the $n+1$ partial sum of the reward process $\left(f\left(X_{n}\right) ; n \in \mathbb{N}\right)$.

- $\mu=\sum_{i \in E} f(i) \pi(i)=\mathbb{E}_{\pi} f\left(X_{0}\right) ; \mu$ indicates the expectation of $f\left(X_{0}\right)$ with respect to the stationary distribution of $\mathbf{X}$.

- $\tilde{f}(i)=f(i)-\mu ; \tilde{f}$ will denote $f$ centralized with respect to the stationary distribution of $\mathbf{X}$.

- $\boldsymbol{e}_{i}$ are the vectors from the orthonormal base of $\mathbb{R}^{s}$.

- $T_{a}=\inf \left\{n>0: X_{n}=a\right\}, a \in E ; T_{a}$ records the first hitting time of state $a$ disregarding the initial state.

- $\mathbb{P}_{i}(A)=\mathbb{P}\left(A \mid X_{0}=i\right)$ where $A \in \mathscr{F}$ (i.e., A represents a measurable event), 
$\mathbb{E}_{i}$ the corresponding expectation operator, $i \in E$.

- $g_{a}(i, j)=\sum_{n=1}^{+\infty} \mathbb{P}_{i}\left(T_{a} \geq n, X_{n}=j\right)$.

- $P_{a}$ is a $(s-1) \times(s-1)$ matrix which results from $P$ excluding both its $a$-column and $a$-row.

- $P_{a}^{\prime}$ is a $s \times(s-1)$ matrix which results from $P$ excluding only his $a$-column.

- $\mathbf{1}$ is an s-dimensional row vector, having all his elements equal to 1.

- $A=\mathbf{1}^{\top} \mathbf{1}$ and $I$ denotes the s-dimensional identity matrix.

Note that,

$$
\begin{aligned}
g_{a}(i, j) & =\sum_{n=1}^{+\infty} \mathbb{E}\left(1\left\{T_{a} \geq n, X_{n}=j\right\} \mid X_{0}=i\right) \\
& =\mathbb{E}\left(\sum_{n=1}^{+\infty} 1\left\{T_{a} \geq n, X_{n}=j\right\} \mid X_{0}=i\right)=\mathbb{E}_{i}\left(N_{j}^{a}\right),
\end{aligned}
$$

where, $N_{j}^{a}$ is a r.v. which counts, after the system leaves from the initial state, how many times it has visited state $j$, until it reaches for the first time state $a$.

The above expression of $g_{a}(i, j)$ gives a concrete probabilistic meaning to this quantity and should be distinguished from, $g_{a}^{*}(i, j)=\sum_{n=0}^{+\infty} \mathbb{P}_{i}\left(T_{a} \geq n, X_{n}=\right.$ $j$ ), which is often used in the literature and will be mentioned also later in this paper. It is obvious that for $a, i, j \in E$,

$$
g_{a}^{*}(i, j)=\delta_{i j}+g_{a}(i, j)
$$

The function $g_{a}(i, j)$ will play a fundamental role in the analysis of the variance on the CLT. Equivalent representations of this variance already exist, and we will indicate some of them in matrix form. These forms can be expressed as a function of the transition probability matrix $P$ of the $\mathrm{MC}$ together with the reward function $f$. Therefore, all these forms can serve as plug-in type estimators for the variance. For the problem of estimation we will choose 
one form involving the notion of hitting times. By proving the asymptotic normality we gain different representations of the asymptotic variance of these estimators. The following theorem is the starting point of our work.

Theorem 1 (Port (1994)) Assume that for some state $a \in E$, $\sum_{i, j \in E} \pi(i) g_{a}(i, j)|\tilde{f}(i)||\tilde{f}(j)|<\infty$ and set

$$
\sigma^{2}=\sum_{i \in E} \pi(i) \tilde{f}(i)^{2}+2 \sum_{\substack{i \neq a \\ i, j \in E}} \pi(i) g_{a}(i, j) \tilde{f}(i) \tilde{f}(j)
$$

Then for any initial distribution, $(1 / \sqrt{n})\left(S_{n}-n \mu\right) \stackrel{d}{\rightarrow} \sigma Z$ where $Z$ is standard normally distributed. Here $E=\mathbb{N}$.

For a proof of this theorem see (Port (1994)).

Remark. It is also proved easily (see Port (1994)) that the validity of the condition stated in Theorem 1 does not depend on the fixed state $a$ that we choose each time.

\section{Variance calculation and equivalent matrix forms}

The quantities $g_{a}(i, j)$, which appear in Theorem 1 , involve both the state of the system at time $n$ and the first return time to a fixed state $a$. We can further analyze them and get a representation in terms of the transition matrix in the case of finite state space. The following proposition serves this purpose. We use $I_{a}$ for the (s-1)-dimensional identity matrix, since as we did for $P$ to get $P_{a}$, the matrix $I_{a}$ results from $I$ excluding both its $a$-column and $a$-row.

Proposition 1 Let $\boldsymbol{X}$ be a $M C$, having finite state space $E=\{1, \ldots, s\}$ and 
transition matrix P. Fix $a \in E$. Then,

$$
g_{a}(i, j)= \begin{cases}\boldsymbol{e}_{i} P_{a}^{\prime}\left(I_{a}-P_{a}\right)^{-1} \boldsymbol{e}_{j^{\prime}}^{\top} & \text { if } j \neq a, \\ 1 & \text { if } j=a,\end{cases}
$$

where $j^{\prime}=j$ for $j<a$, while $j^{\prime}=j-1$ for $j>a, i, j \in E$.

PROOF. For $j \neq a$,

$$
\begin{aligned}
& \mathbb{P}_{i}\left(T_{a} \geq n, X_{n}=j\right) \\
& =\sum_{i_{1}} \sum_{i_{2}} \ldots \sum_{i_{n-1}} \mathbb{P}_{i}\left(X_{1}=i_{1}, X_{2}=i_{2}, \ldots, X_{n-1}=i_{n-1}, X_{n}=j, T_{a} \geq n\right) \\
& =\sum_{i_{1} \neq a} \sum_{i_{2} \neq a} \ldots \sum_{i_{n-1} \neq a} \mathbb{P}_{i}\left(X_{1}=i_{1}, X_{2}=i_{2}, \ldots, X_{n-1}=i_{n-1}, X_{n}=j\right) \\
& =\sum_{i_{1} \neq a} \sum_{i_{2} \neq a} \ldots \sum_{i_{n-1} \neq a} \mathbb{P}\left(i, i_{1}\right) \mathbb{P}\left(i_{1}, i_{2}\right) \ldots \mathbb{P}\left(i_{n-1}, j\right)=\boldsymbol{e}_{i} P_{a}^{\prime} P_{a}^{n-1} \boldsymbol{e}_{j^{\prime}}^{\top}
\end{aligned}
$$

Consequently, using the definition of $g_{a}(i, j)$ and relation (4), we have

$$
\begin{aligned}
g_{a}(i, j) & =\sum_{n=1}^{+\infty} \mathbb{P}_{i}\left(T_{a} \geq n, X_{n}=j\right)=\sum_{n=1}^{+\infty} \boldsymbol{e}_{i} P_{a}^{\prime} P_{a}^{n-1} \boldsymbol{e}_{j^{\prime}}^{\top} \\
& =\boldsymbol{e}_{i} P_{a}^{\prime}\left(\sum_{n=1}^{+\infty} P_{a}^{n-1}\right) \boldsymbol{e}_{j^{\prime}}^{\top}=\boldsymbol{e}_{i} P_{a}^{\prime}\left(I_{a}-P_{a}\right)^{-1} \boldsymbol{e}_{j^{\prime}}^{\top}
\end{aligned}
$$

The last equality is valid since the series $\sum_{n=1}^{+\infty} P_{a}^{n-1}$ is convergent. This follows from the fact that $P$ is irreducible and, after excluding the state $a$, the remaining matrix $P_{a}$ has spectral radius strictly less than 1 . For a nice presentation of the spectral analysis for MC see Seneta (2006).

When $j=a$,

$$
\mathbb{P}_{i}\left(T_{a} \geq n, X_{n}=j\right)=\mathbb{P}_{i}\left(T_{a} \geq n, X_{n}=a\right)=\mathbb{P}_{i}\left(T_{a}=n\right)
$$


Therefore,

$$
g_{a}(i, j)=g_{a}(i, a)=\sum_{n=1}^{\infty} \mathbb{P}_{i}\left(T_{a}=n\right)=\mathbb{P}_{i}\left(T_{a}<\infty\right)=1
$$

We can easily gain an expression of the stationary distribution $\boldsymbol{\pi}$ in terms of the transition matrix by using its definition.

$\boldsymbol{\pi}=\boldsymbol{\pi} P$, so $\boldsymbol{\pi}(I-P)=\mathbf{0}$ and then $\boldsymbol{\pi}(I-P)+\mathbf{1}=\mathbf{1}$.

Finally, since $\boldsymbol{\pi} A=\mathbf{1}$, we conclude that

$$
\boldsymbol{\pi}=\mathbf{1}(I-P+A)^{-1}
$$

Remark. The matrix $I-P+A$ is non singular and a proof of this fact can be found in Resnick (1992), Proposition 2.14.1. The non singularity depends on the uniqueness of the stationary distribution. His treatment involves the irreducible chains but his proof can be extended to include transient states too. The invertibility of $I-P+A$ turns out to be a characterization of the finite Markov chains with exactly one positive recurrent class, or equivalently, with a unique stationary distribution, where the zeros in the stationary distribution indicate, as it is well known, the transient states.

We are going to use the following notation in order to have a closed matrix form for $\sigma^{2}$, including all information from the transition matrix.

- $\boldsymbol{\pi}^{*}=(\pi(1) \tilde{f}(1) \quad \pi(2) \tilde{f}(2) \ldots \pi(s) \tilde{f}(s))$.

- $D=\left(d_{i j}\right)$, where $d_{i j}=g_{a}(i, j)$ if $i \neq a$ and $d_{a, j}=0, j \in E$.

- $\Pi=\mathbf{1}^{\top} \boldsymbol{\pi}$.

The matrix $\Pi$ is the Cesaro limit of the sequence $\left\{P^{n} ; n \geq 0\right\}$ and, in particular, the limiting matrix, if we strengthen the condition of irreducibility (in the 
finite case) with aperiodicity. By $\Pi_{d g}$ we denote as the matrix which results from $\Pi$ by putting all the off-diagonal elements equal to zero.

By using relation (6), we have the following expression of $\Pi$.

$$
\Pi=A(I-P+A)^{-1} .
$$

From (3) we get

$$
\sigma^{2}=\boldsymbol{\pi}^{*} \tilde{\boldsymbol{f}}^{\top}+2 \boldsymbol{\pi}^{*} D \tilde{\boldsymbol{f}}^{\top}=\boldsymbol{\pi}^{*}(I+2 D) \tilde{\boldsymbol{f}}^{\top}
$$

By analyzing expression (8), one can get the desired result for $\sigma^{2}$ in a matrix form as a function of $P$. The vector $\pi^{*}$ can be written as follows $\boldsymbol{\pi}^{*}=\boldsymbol{\pi} \widetilde{F}=\boldsymbol{\pi}\left[F-\left(\boldsymbol{\pi} \boldsymbol{f}^{\top}\right) I\right]$, where $\widetilde{F}=\operatorname{diag}\{\widetilde{f}(i), i \in S\}, \mathrm{F}=\operatorname{diag}\{f(i), i \in$ $E\}$.

So, by using expression (6), we have the following form

$$
\boldsymbol{\pi}^{*}=\mathbf{1}(I-P+A)^{-1}\left[F-\left(\mathbf{1}(I-P+A)^{-1} \boldsymbol{f}^{\top}\right) I\right] .
$$

Next, we introduce two more constant matrices. Firstly, we denote by $B$ a $s \times(s-1)$ matrix that results from a $s-1$ order identity matrix, by including a null row between his $a-1$ and $a$ row. Its use combined with his transpose is to expand a given $s-1$ dimension matrix $M$ to a $s$ dimension one, having its $a$ row and its $a$ column null. If we have a vice versa use on a $s$ dimension matrix $M$ i.e., first by multiplying from the left with the transpose of $B$ then multiplying from the right with $B$, we delete its $a$ row and its $a$ column. Secondly, we denote by $\mathrm{C}$ the matrix who results from the null matrix, replacing its $a$ column with the vector $\left[\begin{array}{llllllll}1 & 1 & \ldots & 1 & 0 & 1 & \ldots & 1\end{array}\right]^{\top}$ where 0 occupies the $a$-th place.

Remark. Since D depends only on $g_{a}(i, j)$ for $i \neq a$, we can have the expression 
$g_{a}(i, j)=\boldsymbol{e}_{i^{\prime}} P_{a}\left(I_{a}-P_{a}\right)^{-1} \boldsymbol{e}_{j^{\prime}}^{\top}$, where $i^{\prime}=i-1_{(i>a)}, j^{\prime}=j-1_{(j>a)}$, for $i, j \neq a$.

Using the above notation,

$$
\begin{aligned}
D & =B\left[P_{a}\left(I_{a}-P_{a}\right)^{-1}\right] B^{\top}+C=B\left[B^{\top} P B\left(I_{a}-B^{\top} P B\right)^{-1}\right] B^{\top}+C, \\
\tilde{\boldsymbol{f}}^{\top} & =\boldsymbol{f}^{\top}-\left(\boldsymbol{\pi} \boldsymbol{f}^{\top}\right) \mathbf{1}^{\top}=\boldsymbol{f}^{\top}-\left[\mathbf{1}(I-P+A)^{-1} \boldsymbol{f}^{\top}\right] \mathbf{1}^{\top} .
\end{aligned}
$$

From (1) we can see that the elements $D_{i j}$ of the matrix $D$ express the mean total number of visits to state $j$, starting from state $i$, until it reaches for the first time state $a$. The count does not include the initial state and also state $a$ is not allowed as an initial state (all the elements corresponding to a-row are zero). The vector $\tilde{\boldsymbol{f}}^{\top}$ results from the values of $f$ if we subtract from each value the expectation of $f(X)$, when $X$ follows the stationary distribution of the MC X. Using relations (9), (10) and (11), we can conclude that for a given function $f, \sigma^{2}$ is a function of $P$ expressed in a compact form by relation (8).

Different representations of $\sigma^{2}$ as a function of $P$ lead to different ways of viewing the MLE of $\sigma^{2}$. In the bibliography, it can be found in the following form (see Doob (1953), Lemma 7.3 or Billingsley (1961b), Theorem 3.3, remarks)

$$
\sigma^{2}=E\left\{\tilde{f}\left(X_{0}\right)^{2}\right\}+2 \sum_{k=1}^{+\infty} E\left\{\tilde{f}\left(X_{0}\right) \tilde{f}\left(X_{k}\right)\right\} .
$$

By analyzing relation (12), we get the well known form for $\sigma^{2}$ (see Peskun $(1973))$,

$$
\sigma^{2}=\tilde{\boldsymbol{f}} \Pi_{d g}(2 Z-I) \tilde{\boldsymbol{f}}^{\top}=\boldsymbol{f} \Pi_{d g}(2 Z-I-\Pi) \boldsymbol{f}^{\top}
$$

where $Z=(I-P+\Pi)^{-1}$ is called the fundamental matrix of $P$. A nice description of $Z$ and its properties can be found in Kemeny and Snell (1976). Now, we can present some equal expressions for $\sigma^{2}$.

Proposition 2 Let $X$ be an irreducible $M C$, having finite state space $E=$ 
$\{1, \ldots s\}$. The following expressions for the asymptotic variance $\sigma^{2}$ are equal.

1) $\sigma^{2}=\tilde{\boldsymbol{f}} \Pi_{d g}(2 Z-I) \tilde{\boldsymbol{f}}^{\top}=\boldsymbol{f} \Pi_{d g}(2 Z-I-\Pi) \boldsymbol{f}^{\top}$.

2) $\sigma^{2}=\widetilde{\boldsymbol{f}} \Pi_{d g}\left(2 D^{*}-I\right) \tilde{\boldsymbol{f}}^{\top}=\boldsymbol{f} \Pi_{d g}(I-\Pi)\left(2 D^{*}-I\right)(I-\Pi) \boldsymbol{f}^{\top}$.

$\left.2^{*}\right) \sigma^{2}=\tilde{\boldsymbol{f}} \Pi_{d g}(I+2 D) \tilde{\boldsymbol{f}}^{\top}=\boldsymbol{f} \Pi_{d g}(I-\Pi)(I+2 D)(I-\Pi) \boldsymbol{f}^{\top}=\boldsymbol{\pi}^{*}(I+2 D) \tilde{\boldsymbol{f}}^{\top}$, where $D_{i j}^{*}=g_{a}^{*}(i, j)$ (defined by relation (2)), for $i \neq a$ and $j \neq a$ and $D_{i j}^{*}=0$ for $i=a$ or $j=a$.

PROOF. First, we indicate how, starting from the last form of $2^{*}$ ), which is presented in relation (8), we reach the two other forms of $2^{*}$ ). Analyzing the first factor in relation (8), we get $\boldsymbol{\pi}^{*}=\tilde{\boldsymbol{f}} \Pi_{d g}$ and $\tilde{\boldsymbol{f}}=\boldsymbol{f}\left(I-\Pi^{\top}\right)$. Observe that $\left(I-\Pi^{\top}\right) \Pi_{d g}=\Pi_{d g}(I-\Pi)$, since $\Pi_{d g} \Pi=(\pi(i) \pi(j))_{i, j \in E}$ is a symmetric matrix. Finally, we get

$$
\sigma^{2}=\tilde{\boldsymbol{f}} \Pi_{d g}(I+2 D) \tilde{\boldsymbol{f}}^{\top}=\boldsymbol{f} \Pi_{d g}(I-\Pi)(I+2 D)(I-\Pi) \boldsymbol{f}^{\top}
$$

In order to pass from the forms 1) to their analogues in 2) we need a connection between the matrices $Z$ and $D^{*}$. In Kemeny and Snell (1976), Theorem 6.2.5, it is proved that

$$
Z=\Pi+(I-\Pi) D^{*}(I-\Pi) .
$$

Substituting (15) in the first part of (13) and using that $\Pi \tilde{\boldsymbol{f}}^{\top}=\mathbf{0}^{\top}$, we reach

$$
\sigma^{2}=\tilde{\boldsymbol{f}} \Pi_{d g}\left(2 D^{*}-I\right) \tilde{\boldsymbol{f}}^{\top}=\boldsymbol{f} \Pi_{d g}(I-\Pi)\left(2 D^{*}-I\right)(I-\Pi) \boldsymbol{f}^{\top}
$$

Along the lines of the analysis for $g_{a}(i, j)$ it can be shown easily (see also Kemeny and Snell (1976), pp. 46-47) that

$$
D^{*}=B\left(I_{a}-P_{a}\right)^{-1} B^{\top} .
$$

Since $P_{a}\left(I_{a}-P_{a}\right)^{-1}=\left(I_{a}-P_{a}\right)^{-1}-I_{a}$, we can easily transform the expression of $D$ given from relation (10) in order to have a passage from relation (14) to 
(16).

\section{MLE for finite state space MC}

Let us consider a sample path of a $\mathrm{MC}$ in the time interval $\{0,1, \ldots, \mathrm{m}\}$. The parameter to be estimated is $\boldsymbol{d}=\left(p_{i j}, i, j \in E, i \neq j\right) \in \mathbb{R}^{s^{2}-s}$. We assume that $p_{i j}>0, i, j \in E, i \neq j$. The following notation will be used in the sequel:

- $N_{i}(m)=\sum_{k=0}^{m-1} 1\left\{X_{k}=i\right\} ; N_{i}(m)$ expresses the system's total time spent in state $i$ until time $T=m$.

- $M_{0}=\inf \left\{m \in \mathbb{N}: N_{i}(m)>0, \forall i \in E\right\}$.

- $N_{i j}(m)=\sum_{k=1}^{m} 1\left\{X_{k-1}=i, X_{k}=j\right\}$ and expresses the system's total number of transitions from state $i$ to state $j$ until time $T=m$.

- $\widehat{P}(m)=\left(N_{i j}(m) / N_{i}(m)\right)_{i, j \in E}, \forall m \geq M_{0}$.

- $\boldsymbol{d}(m)=\left(\widehat{p}_{i j}(m), i, j \in E, i \neq j\right)$.

- $M_{. u}=M e_{u}^{\top}$ i.e., the u-th column of any matrix $M$.

- $g=\left(g_{u}, 1 \leq u \leq s\right)$ where $g_{u}=\mathbf{1}(I-P+A)_{. u}^{-1}, 1 \leq u \leq s$, i.e., each component $g_{u}$ regarded as a real function of $s^{2}-s$ variables that $p_{i j}, i, j \in$ $E, i \neq j$ stand for, defining all together an open set in the $s^{2}-s$ Euclidean space and representing our parametric space.

- $H^{i j}=\frac{\partial P}{\partial p_{i j}}(I-P+A)^{-1}$.

Remarks. 1) It is well known, see (Billingsley (1961b)), that the maximum likelihood estimator of $p_{i j}$ is as follows

$\widehat{p}_{i j}(m)=N_{i j}(m) / N_{i}(m), \forall m \geq M_{0}$.

2) $N_{i}(m)=\sum_{j \in E} N_{i j}(m)$.

3) The $s$ diagonal elements of $P$ are determined by the other elements on the same row and the following relation holds: $\widehat{p_{i i}}(m)=1-\sum_{j \in E-\{i\}} \widehat{p_{i j}}(m)$. 
In the following considerations when we use vector notation for the elements of a matrix, we consider them to be ordered taking first the elements of its first row, then the elements of the second, etc.

Proposition 3 Let $X$ be a homogeneous, irreducible finite $M C$. The sequence of random vectors,

$\boldsymbol{\xi}(m)=\left(\xi_{i j}(m), i, j \in E\right)$, where $\xi_{i j}(m)=\sqrt{m}\left(\widehat{p}_{i j}(m)-p_{i j}\right)$,

converges in distribution, as $m$ tends to infinity, to a random vector $\xi$, where $\xi \sim N(\mathbf{0}, \Gamma)$ and $\Gamma$ is a covariance matrix of dimension $s^{2} \times s^{2}$, defined in a block diagonal form

$$
\Gamma=\left(\begin{array}{cccc}
\frac{1}{\pi(1)} \Lambda_{1} & \mathbf{0} & \cdots & \mathbf{0} \\
\mathbf{0} & \frac{1}{\pi(2)} \Lambda_{2} & \cdots & \mathbf{0} \\
\vdots & \vdots & \ddots & \vdots \\
\mathbf{0} & \mathbf{0} & \cdots & \frac{1}{\pi(s)} \Lambda_{s}
\end{array}\right) \text {, where } \Lambda_{i}=\left(p_{i j}\left(\delta_{j l}-p_{i l}\right)\right)_{j, l \in E}, i \in E
$$

For a proof of this proposition see Billingsley (1961b), Theorem 3.1 combined with Sadek and Limnios (2002), Proposition 1.

\subsection{Estimation of the stationary distribution}

We define and compare two estimators of the stationary distribution and establish a relation between them. The first estimator is the MLE for the stationary distribution and it can be derived from (6) by substituting $P$ with its MLE $\widehat{P}(m)$. The second estimator that we define is known as the empirical estimator. We will show that they have the same asymptotic properties. 
(1) $\widehat{\boldsymbol{\pi}}(m):=\mathbf{1}(I-\widehat{P}(m)+A)^{-1} \quad$ (MLE estimator).

The matrix $(I-\widehat{P}(m)+A)^{-1}$ must be well defined. For this to happen, we need to ensure that $I-\widehat{P}(m)+A$ is non singular. Since for $m \geq M_{0}$, all the states of the system and at least one transition for each state have been recorded, $\widehat{P}(m)$ is a stochastic matrix with a unique stationary distribution. Therefore, we can conclude that $I-\widehat{P}(m)+A$ is non singular with probability 1 , for $m \geq M_{0}$.

(2) $\hat{\boldsymbol{\pi}}^{\#}(m):=\left(\frac{N_{j}(m)}{m}\right)_{j \in E} \quad$ (empirical estimator).

This is the very well known estimator which indicates the percentage of time the system has stayed in each of its states.

Before studying the asymptotic properties of these two estimators, we discuss how the estimator $\widehat{\boldsymbol{\pi}}(m)$ behaves for $1 \leq m<M_{0}$. During this period of time $\widehat{P}(m)$ is not a stochastic matrix. Every time that the process visits a new state that has not been recorded yet, even if the matrix $I-\widehat{P}(m)+A$ is non-singular, $\widehat{\boldsymbol{\pi}}(m)$ does not represent a stationary distribution. Another problem occurs when the system stays at this state for a long period of time, since $\widehat{P}(m)$ is recognized as having one positive recurrent state and $\widehat{\boldsymbol{\pi}}(m)$ gives all the weight in this state. The procedure of calculating $\widehat{\boldsymbol{\pi}}(m)$ works well for all the other time points, always for $m<M_{0}$. This follows from an extension of Proposition 2.14.1 in Resnick (1992), to include matrices that can be decomposed to a stochastic submatrix and to zero elements. The stochastic submatrix should correspond to one positive recurrent subclass and possibly some transient states. The proof is similar and it will be omitted. The matrix $\widehat{P}(m)$ can be decomposed to a stochastic submatrix (having as dimension the existing number of visited states), and to zero elements. The states, which correspond to the stochastic submatrix, consist a class with one positive recurrent 
subclass and probably some transient states. Therefore, the matrix operator that we have used for the calculation of the stationary distribution acts in the same way for the submatrix (it calculates the stationary distribution on this subset of visited states), and assigns zeros to non visited states.

Proposition 4 The above two estimators are consistent, that is, i) $\widehat{\boldsymbol{\pi}}(m) \stackrel{\text { a.s. }}{\rightarrow} \boldsymbol{\pi}, \quad$ ii $) \widehat{\boldsymbol{\pi}}^{\#}(m) \stackrel{\text { a.s. }}{\rightarrow} \boldsymbol{\pi}$, as $m \rightarrow \infty$.

PROOF. i) Since $\widehat{p}_{i j}(m) \stackrel{\text { a.s. }}{\rightarrow} p_{i j}$, when $m \rightarrow \infty$, $\widehat{P}(m) \stackrel{\text { a.s. }}{\rightarrow} P$, which implies that $I-\widehat{P}(m)+A \stackrel{\text { a.s. }}{\rightarrow} I-P+A$.

Consequently, $(I-\widehat{P}(m)+A)^{-1} \stackrel{a . s .}{\longrightarrow}(I-P+A)^{-1}$ and then, $\mathbf{1}(I-\widehat{P}(m)+A)^{-1} \stackrel{\text { a.s. }}{\rightarrow} \mathbf{1}(I-P+A)^{-1}$ which gives $\widehat{\boldsymbol{\pi}}(m) \stackrel{\text { a.s. }}{\rightarrow} \boldsymbol{\pi}$.

ii) Since $\frac{N_{j}(m)}{m} \stackrel{a . s .}{\rightarrow} \pi(j)$, for any $j, 1 \leq j \leq s$, a very well known result (see, e.g., Girardin and Limnios (2001)), it is obvious that $\widehat{\boldsymbol{\pi}}^{\#}(m) \stackrel{\text { a.s. }}{\rightarrow} \boldsymbol{\pi}$.

Remark. The proof of Proposition 4 ii) can be inferred from i), if we establish the exact relationship between them. This will be shown later in the sequel from relation (25).

We denote $\Gamma^{*}$ the $\left(s^{2}-s\right) \times\left(s^{2}-s\right)$ matrix who results from $\Gamma$ excluding his $(s+1) i+1, i=0, \ldots, s-1$ order rows and columns.

Proposition 5 The MLE of the stationary distribution is asymptotically normal, that is, $\sqrt{m}(\widehat{\boldsymbol{\pi}}(m)-\boldsymbol{\pi}) \stackrel{d}{\rightarrow} N\left(\mathbf{0}, G \Gamma^{*} G^{\top}\right)$, where

$$
G=\left(\partial g_{u} / \partial p_{i j}\right)_{s \times\left(s^{2}-s\right)}, u, i, j \in E, i \neq j
$$


and

$$
\frac{\partial g_{u}}{\partial p_{i j}}=\pi(j)(I-P+A)_{j u}^{-1}-\pi(i)(I-P+A)_{i u}^{-1}, u, i, j \in E, i \neq j
$$

PROOF. We have

$$
\begin{aligned}
\sqrt{m}(\widehat{\boldsymbol{\pi}}(m)-\boldsymbol{\pi}) & =\sqrt{m}\left[\mathbf{1}(I-\widehat{P}(m)+A)^{-1}-\mathbf{1}(I-P+A)^{-1}\right] \\
& =\sqrt{m}[g(\boldsymbol{d}(m))-g(\boldsymbol{d})] .
\end{aligned}
$$

Since, $\underline{\xi}(m) \stackrel{d}{\rightarrow} N(\mathbf{0}, \Gamma)$ then, $\sqrt{m}(\boldsymbol{d}(m)-\boldsymbol{d}) \stackrel{d}{\rightarrow} N\left(\mathbf{0}, \Gamma^{*}\right)$, as it is a marginal distribution of multivariate normal.

We want to conclude that, $\sqrt{m}[g(\boldsymbol{d}(m))-g(\boldsymbol{d})] \stackrel{d}{\rightarrow} N\left(\mathbf{0}, G \Gamma^{*} G^{\top}\right)$.

The last relation will follow from delta method, if $g$ has components with continuous first order partial derivatives. In our case we can easily conclude this, since the components of $g$ depend on the elements of $(I-P+A)^{-1}$. Indeed, every element of this matrix is a ratio of polynomials (a rational function) of $p_{i j}$, where the denominator is the determinant of $I-P+A$ and it cannot be zero by assumption. So we have existence and continuity of partial derivatives. Their exact values are:

$$
\begin{aligned}
\frac{\partial g_{u}}{\partial p_{i j}} & =\frac{\partial \mathbf{1}(I-P+A)_{\cdot u}^{-1}}{\partial p_{i j}}=\frac{\partial \mathbf{1}(I-P+A)^{-1} \boldsymbol{e}_{u}^{\top}}{\partial p_{i j}} \\
& =\mathbf{1}(I-P+A)^{-1} \frac{\partial P}{\partial p_{i j}}(I-P+A)^{-1} \boldsymbol{e}_{u}^{\top} \\
& =\boldsymbol{\pi} \frac{\partial P}{\partial p_{i j}}(I-P+A)^{-1} \boldsymbol{e}_{u}^{\top}=\boldsymbol{\pi} \frac{\partial P}{\partial p_{i j}}(I-P+A)_{. u}^{-1}
\end{aligned}
$$

The $s \times s$ dimensional matrix $\partial P / \partial p_{i j}$ has all its elements zero except for its (i,j)-element being 1 and its (i,i)-element being -1. So, from relation (20) we conclude (19).

Remark. In the previous analysis we assumed that at least $s^{2}-s$ elements of $P$ were strictly positive by taking exactly $s^{2}-s$ variables. These may be 
less by properly estimating the set of possible transitions. By reducing the variables of $P$, we can follow the same steps, adjust matrix dimensions, take the proper covariance matrix and reach the same conclusion.

In a similar way as it is done for $N_{i}(m)$, we denote

- $N_{j}^{*}(m)=\sum_{k=1}^{m} 1\left\{X_{k}=j\right\}=N_{j}(m)+\delta_{j X_{m}}-\delta_{j X_{0}}$.

Proposition 6 The empirical estimator of the stationary distribution is asymptotically normal, that is, $\sqrt{m}\left(\widehat{\boldsymbol{\pi}}^{\#}(m)-\boldsymbol{\pi}\right) \stackrel{d}{\rightarrow} N\left(\mathbf{0}, G \Gamma^{*} G^{\top}\right)$, as $m \rightarrow \infty$.

PROOF. We will show in fact the exact relation between $\widehat{\boldsymbol{\pi}}^{\#}(m)$ and $\widehat{\boldsymbol{\pi}}(m)$.

We simply notice that

$$
\widehat{\pi}^{\#}(m) \widehat{P}(m)=\left(\frac{N_{1}(m)}{m} \frac{N_{2}(m)}{m} \ldots \frac{N_{s}(m)}{m}\right)\left(\begin{array}{ccc}
\frac{N_{11}(m)}{N_{1}(m)} & \cdots & \frac{N_{1 s}(m)}{N_{1}(m)} \\
\frac{N_{21}(m)}{N_{2}(m)} & \cdots & \frac{N_{2 s}(m)}{N_{2}(m)} \\
\vdots & \ddots & \vdots \\
\frac{N_{s 1}(m)}{N_{s}(m)} & \cdots & \frac{N_{s s}(m)}{N_{s}(m)}
\end{array}\right),
$$

which implies that

$$
\widehat{\boldsymbol{\pi}}^{\#}(m) \widehat{P}(m)=\left(\frac{\sum_{i=1}^{s} N_{i 1}(m)}{m} \frac{\sum_{i=1}^{s} N_{i 2}(m)}{m} \ldots \frac{\sum_{i=1}^{s} N_{i s}(m)}{m}\right)
$$

As $\widehat{\boldsymbol{\pi}}(m)=\mathbf{1}(I-\widehat{P}(m)+A)^{-1}$, for any $m \geq M_{0}$, we have that $\widehat{\boldsymbol{\pi}}(m)$ is the unique stationary distribution of $\widehat{P}(m)$. Now, we will examine if $\widehat{\pi}^{\#}(m)$ can play this role for some $m$. By Equation (21), this holds if

$$
N_{j}(m)=\sum_{i=1}^{s} N_{i j}(m), \forall j, 1 \leq j \leq s
$$


It is obvious that

$$
N_{j}^{*}(m)=\sum_{i=1}^{s} N_{i j}(m) .
$$

Using the definition of $N_{j}^{*}(m)$ and relation (23), we conclude from (21) that

$$
\widehat{\boldsymbol{\pi}}^{\#}(m) \widehat{P}(m)=\widehat{\boldsymbol{\pi}}^{\#}(m)+\boldsymbol{\Delta}(m)
$$

where $\Delta_{i}(m)=\left(\delta_{i X_{m}}-\delta_{i X_{0}}\right) / m, i \in E$.

We take the path $\left(X_{0} X_{1} \ldots X_{m}\right)$ and, as $m \rightarrow \infty$, we observe that every time $X_{m}=X_{0}$, we have $\Delta_{i}(m)=0$, for any $i \in E$. In this way $\widehat{\pi}^{\#}(m)$ satisfies the balance equation of $\widehat{P}(m)$ and therefore coincides with $\widehat{\boldsymbol{\pi}}(m)$ by defining a common subsequence indexed by the random times that the system visits the initial state. Analytically, their relationship can be written as follows.

From (24), we get

$\widehat{\boldsymbol{\pi}}^{\#}(m)(I-\widehat{P}(m)+A)=\mathbf{1}-\boldsymbol{\Delta}(m)$.

Thus, we have

$$
\begin{aligned}
\widehat{\boldsymbol{\pi}}^{\#}(m) & =(\mathbf{1}-\boldsymbol{\Delta}(m))(I-\widehat{P}(m)+A)^{-1} \\
& =\widehat{\boldsymbol{\pi}}(m)-\boldsymbol{\Delta}(m)(I-\widehat{P}(m)+A)^{-1} .
\end{aligned}
$$

Using relation $(25)$, we get

$$
\sqrt{m}\left(\widehat{\boldsymbol{\pi}}^{\#}(m)-\boldsymbol{\pi}\right)=\sqrt{m}(\widehat{\boldsymbol{\pi}}(m)-\boldsymbol{\pi})-\sqrt{m} \boldsymbol{\Delta}(m)(I-\widehat{P}(m)+A)^{-1} .
$$

But, $\max _{1 \leq i \leq s} \sqrt{m}\left|\Delta_{i}(m)\right| \leq 1 / \sqrt{m}$ which gives $\sqrt{m} \boldsymbol{\Delta}(m) \stackrel{\text { a.s. }}{\rightarrow} \mathbf{0}$.

Since $(I-\widehat{P}(m)+A)^{-1} \stackrel{a . s .}{\rightarrow}(I-P+A)^{-1}$,

we conclude that

$$
\sqrt{m} \boldsymbol{\Delta}(m)(I-\widehat{P}(m)+A)^{-1} \stackrel{a . s .}{\rightarrow} \mathbf{0}
$$

With the aid of relation (27) and Proposition 5, using Slutsky Theorem, we conclude from Equation $(26)$ that $\sqrt{m}\left(\widehat{\boldsymbol{\pi}}^{\#}(m)-\boldsymbol{\pi}\right) \stackrel{d}{\rightarrow} N\left(\mathbf{0}, G \Gamma^{*} G^{\top}\right)$. 


\subsection{MLE for the Variance $\sigma^{2}$}

The problem of estimation when the transition matrix is unknown will be our next goal. With the aid of (7) and (17), all the equivalent expressions given in Proposition 2 can be written as functions of the transition matrix and, therefore, serve as representations for the MLE of $\sigma^{2}$ if $P$ is substituted by its MLE $\widehat{P}$. We study the representation which is defined from Equation (3) and expressed in terms of the transition matrix from Equation (8). We will prove that it is strongly consistent and asymptotically normal. Additionally, we will find its asymptotic variance. By observing one trajectory in the time interval $\{0,1, \ldots, \mathrm{m}\}$ we define

$$
\widehat{\sigma^{2}}(m)=\widehat{\boldsymbol{\pi}^{*}}(m)(I+2 \widehat{D}(m)) \widehat{\widetilde{\boldsymbol{f}}}^{\top}(m)
$$

where $\widehat{\boldsymbol{\pi}^{*}}(m), \widehat{D}(m), \widehat{\widetilde{\boldsymbol{f}}}(m)$ are estimators of $\boldsymbol{\pi}^{*}, D, \tilde{\boldsymbol{f}}$ which are defined by Equations (9), (10) and (11), and for a given function f, they depend only on the probability matrix. So, by taking as an estimator of $P$ the one defined on Section 3, substituting $P$ in the Equations $(9),(10),(11)$ by $\widehat{P}(m)$ we get the above estimators.

Consider $P$ to be a function of $s^{2}-s$ variables, denoted by $\boldsymbol{d}$ (see previous Section) and, set $\sigma^{2}=\Phi(\boldsymbol{d})=\Phi\left(p_{i j}, i, j \in E, i \neq j\right)$.

Theorem 2 Let $\sigma^{2}$ defined as in Theorem 1 and $\widehat{\sigma^{2}}(m)$ the MLE of $\sigma^{2}$ represented in the form $\widehat{\boldsymbol{\pi}}^{*}(m)(I+2 \widehat{D}(m)) \widehat{\widetilde{\boldsymbol{f}}}^{\top}(m)$. Then, the following asymptotic results hold

i) $\widehat{\sigma^{2}}(m) \stackrel{\text { a.s. }}{\rightarrow} \sigma^{2}$,

ii) If $\sigma^{2}>0$ then $\sqrt{m}\left(\widehat{\sigma^{2}}(m)-\sigma^{2}\right) \stackrel{d}{\rightarrow} N\left(0, \Phi^{\prime} \Gamma^{*}\left(\Phi^{\prime}\right)^{\top}\right)$, where $\Phi^{\prime}=\left[\partial \Phi / \partial p_{i j}\right]_{1 \times\left(k^{2}-k\right)}$ and $\Gamma^{*}$ defined before Proposition 5 . 
The elements of the matrix $\Phi^{\prime}$ are described as follows

$$
\begin{aligned}
\frac{\partial \Phi}{\partial p_{i j}}= & \boldsymbol{\pi}\left[H^{i j} \widetilde{F}-\left(\boldsymbol{\pi} H^{i j} \boldsymbol{f}^{\top}\right) I\right](I+2 D) \tilde{\boldsymbol{f}}^{\top}+ \\
& +2 \boldsymbol{\pi}^{*} B\left[I_{a}+P_{a}\left(I_{a}-P_{a}\right)^{-1}\right]\left[B^{\top} \frac{\partial P}{\partial p_{i j}} B\left(I_{a}-P_{a}\right)^{-1}\right] B^{\top} \tilde{\boldsymbol{f}}^{\top}- \\
& -\boldsymbol{\pi}^{*}(I+2 D)\left(\boldsymbol{\pi} H^{i j} \boldsymbol{f}^{\top}\right) \mathbf{1}^{\top} .
\end{aligned}
$$

PROOF. i) We have:

$$
\begin{aligned}
\widehat{\boldsymbol{\pi}^{*}}(m) & =\mathbf{1}(I-\widehat{P}(m)+A)^{-1}\left[F-\left(\mathbf{1}(I-\widehat{P}(m)+A)^{-1} \boldsymbol{f}^{\top}\right) I\right], \\
\widehat{D}(m) & =B\left[B^{\top} \widehat{P}(m) B\left(I_{a}-B^{\top} \widehat{P}(m) B\right)^{-1}\right] B^{\top}+C, \\
\widehat{\widetilde{\boldsymbol{f}}}^{\top}(m) & =\boldsymbol{f}^{\top}-\left[\mathbf{1}(I-\widehat{P}+A)^{-1} \boldsymbol{f}^{\top}\right] \mathbf{1}^{\top} .
\end{aligned}
$$

Note that multiplying and adding matrices or taking the inverse of a nonsingular matrix are continuous operations. Therefore, using the strong consistency of $\widehat{P}(m)$ and the continuous mapping theorem, we conclude from Equations $(30),(31)$ and (32) that $\widehat{\boldsymbol{\pi}^{*}}(m) \stackrel{\text { a.s }}{\rightarrow} \boldsymbol{\pi}^{*}, \widehat{D}(m) \stackrel{\text { a.s }}{\rightarrow} D, \widehat{\widetilde{\boldsymbol{f}}}^{\top}(m) \stackrel{\text { a.s }}{\rightarrow} \tilde{\boldsymbol{f}}^{\top}$. By combining these results we conclude from Slutsky Theorem that $\widehat{\sigma^{2}}(m)=\widehat{\boldsymbol{\pi}^{*}}(m)(I+2 \widehat{D}(m)) \widehat{\widetilde{\boldsymbol{f}}}^{\top}(m) \stackrel{a . s}{\rightarrow} \boldsymbol{\pi}^{*}(I+2 D) \widetilde{\boldsymbol{f}}^{\top}=\sigma^{2}$

ii) We need to ensure that $\Phi$ has continuous first order partial derivatives. This will be the case if every factor in the factorization of $\sigma^{2}$ in relation (8), considered as a function of $\boldsymbol{d}$, has continuous first order partial derivatives. Take the definition of the first factor $\boldsymbol{\pi}^{*}=(\pi(1) \tilde{f}(1) \quad \pi(2) \tilde{f}(2) \ldots \pi(s) \tilde{f}(s))$. From the previous Section we have concluded that every component of $\boldsymbol{\pi}$ is a ratio of polynomials of $p_{i j}$. Since $\tilde{f}(i)=f(i)-\mu=f(i)-\sum_{i \in E} f(i) \pi(i)$ is also a ratio of polynomials of $p_{i j}$ with denominator $\operatorname{det}(I-P+A)$, we get that every component of $\boldsymbol{\pi}^{*}$ is a ratio of polynomials of $p_{i j}$ (always the admissible ones) with denominator $\operatorname{det}^{2}(I-P+A)$. Thus, the desired result follows. With 
similar arguments we can reach the conclusion that $I+D$ regarded as a vector consists of components which have continuous first order partial derivatives. In this case the denominator is $\operatorname{det}\left(I_{a}-P_{a}\right)$. The same holds for $\widetilde{\boldsymbol{f}}^{\top}$ with denominator $\operatorname{det}(I-P+A)$.

After this discussion we will implement the delta method (see, e.g. Chapter 3, Van Der Vaart (1998)). We have

$\sqrt{m}\left(\widehat{\sigma^{2}}(m)-\sigma^{2}\right)=\sqrt{m}[\Phi(\boldsymbol{d}(m))-\Phi(\boldsymbol{d})]$,

and since

$\sqrt{m}(\boldsymbol{d}(m)-\boldsymbol{d}) \stackrel{d}{\rightarrow} N\left(\mathbf{0}, \Gamma^{*}\right)$,

we conclude that,

$\sqrt{m}[\Phi(\boldsymbol{d}(m))-\Phi(\boldsymbol{d})] \stackrel{d}{\rightarrow} N\left(\mathbf{0}, \Phi^{\prime} \Gamma^{*}\left(\Phi^{\prime}\right)^{\top}\right)$.

Let us calculate $\partial \Phi / \partial p_{i j}$ by using relation (8), that is, from the fact that $\sigma^{2}=\Phi(\boldsymbol{d})=\boldsymbol{\pi}^{*}(I+2 D) \tilde{\boldsymbol{f}}^{\top}$

we get

$$
\frac{\partial \Phi}{\partial p_{i j}}=\frac{\partial \boldsymbol{\pi}^{*}}{\partial p_{i j}}(I+2 D) \tilde{\boldsymbol{f}}^{\top}+2 \boldsymbol{\pi}^{*} \frac{\partial D}{\partial p_{i j}} \tilde{\boldsymbol{f}}^{\top}+\boldsymbol{\pi}^{*}(I+2 D) \frac{\partial \widetilde{\boldsymbol{f}}^{\top}}{\partial p_{i j}}
$$

We analyze further the derivatives which appear in the above Equation.

$$
\frac{\partial \boldsymbol{\pi}^{*}}{\partial p_{i j}}=\frac{\partial \boldsymbol{\pi} \widetilde{F}}{\partial p_{i j}}=\frac{\partial \boldsymbol{\pi}}{\partial p_{i j}} \widetilde{F}+\boldsymbol{\pi} \frac{\partial \widetilde{F}}{\partial p_{i j}} .
$$

Now, using that

$$
\frac{\partial \boldsymbol{\pi}}{\partial p_{i j}}=\frac{\partial \mathbf{1}(I-P+A)^{-1}}{\partial p_{i j}}=\mathbf{1}(I-P+A)^{-1} \frac{\partial P}{\partial p_{i j}}(I-P+A)^{-1}
$$

we conclude that

$$
\frac{\partial \boldsymbol{\pi}}{\partial p_{i j}}=\boldsymbol{\pi} H^{i j}
$$

Also, 


$$
\begin{aligned}
& \frac{\partial \widetilde{F}}{\partial p_{i j}}=\frac{\partial\left(F-\left(\boldsymbol{\pi} \boldsymbol{f}^{\top}\right) I\right)}{\partial p_{i j}}=-\left(\boldsymbol{\pi} H^{i j} \boldsymbol{f}^{\top}\right) I \\
& \frac{\partial D}{\partial p_{i j}}=\frac{\partial\left(B\left[P_{a}\left(I_{a}-P_{a}\right)^{-1}\right] B^{\top}+C\right)}{\partial p_{i j}}=B \frac{\partial\left[P_{a}\left(I_{a}-P_{a}\right)^{-1}\right]}{\partial p_{i j}} B^{\top} .
\end{aligned}
$$

Furthermore,

$$
\begin{aligned}
\frac{\partial\left[P_{a}\left(I_{a}-P_{a}\right)^{-1}\right]}{\partial p_{i j}} & =\frac{\partial P_{a}}{\partial p_{i j}}\left(I_{a}-P_{a}\right)^{-1}+P_{a} \frac{\partial\left(I_{a}-P_{a}\right)^{-1}}{\partial p_{i j}}= \\
& =\frac{\partial P_{a}}{\partial p_{i j}}\left(I_{a}-P_{a}\right)^{-1}+P_{a}\left(I_{a}-P_{a}\right)^{-1} \frac{\partial P_{a}}{\partial p_{i j}}\left(I_{a}-P_{a}\right)^{-1}
\end{aligned}
$$

By substituting the derivative, we get

$$
\frac{\partial\left[P_{a}\left(I_{a}-P_{a}\right)^{-1}\right]}{\partial p_{i j}}=\left[I_{a}+P_{a}\left(I_{a}-P_{a}\right)^{-1}\right]\left[B^{\top} \frac{\partial P}{\partial p_{i j}} B\left(I_{a}-P_{a}\right)^{-1}\right]
$$

Using relations (35), and (36), we conclude from relation (34) that

$$
\frac{\partial \boldsymbol{\pi}^{*}}{\partial p_{i j}}=\boldsymbol{\pi}\left[H^{i j} \widetilde{F}-\left(\boldsymbol{\pi} H^{i j} \boldsymbol{f}^{\top}\right) I\right]
$$

Using relation (38), relation (37) gives us

$$
\frac{\partial D}{\partial p_{i j}}=B\left[I_{a}+P_{a}\left(I_{a}-P_{a}\right)^{-1}\right]\left[B^{\top} \frac{\partial P}{\partial p_{i j}} B\left(I_{a}-P_{a}\right)^{-1}\right] B^{\top}
$$

Also,

$$
\frac{\partial \tilde{\boldsymbol{f}}^{\top}}{\partial p_{i j}}=-\left(\boldsymbol{\pi} H^{i j} \boldsymbol{f}^{\top}\right) \mathbf{1}^{\top}
$$

Now, the final expression for $\partial \Phi / \partial p_{i j}$ is given by inserting in (33), the expressions found in (39), (40) and (41).

Remark. In the previous analysis, the number of variables we used was $s^{2}-s$, assuming that their values are strictly positive. As mentioned in the previous 
Section too, we can reach the same conclusions with fewer positive variables (if this is the case), by modifying properly the vector and matrix dimensions.

By Proposition 2, using all these forms, we can have equivalent representations of $\partial \Phi / \partial p_{i j}$. Since forms 2) and 2)* are similar, it is worth studying form 1) involving the fundamental matrix of $P$.

Proposition 7 The elements of $\Phi^{\prime}$ described in Theorem 2 can be written in the form

$$
\frac{\partial \Phi}{\partial p_{i j}}=\boldsymbol{f}\left(\left(\Pi H^{i j}\right)_{d g}(2 Z-I-\Pi)+\Pi_{d g}\left(2 Z\left(\frac{\partial P}{\partial p_{i j}}-\Pi H^{i j}\right) Z-\Pi H^{i j}\right)\right) \boldsymbol{f}^{\top}
$$

PROOF. Similarly to relation (35), we have

$$
\frac{\partial \Pi}{\partial p_{i j}}=\Pi \frac{\partial P}{\partial p_{i j}}(I-P+A)^{-1}=\Pi H^{i j}
$$

Viewing $Z$ as a function of the non diagonal elements of $P$ we conclude for $i, j \in E, i \neq j$, that

$$
\begin{aligned}
\frac{\partial Z}{\partial p_{i j}} & =-(I-P+\Pi)^{-1}\left(-\frac{\partial P}{\partial p_{i j}}+\frac{\partial \Pi}{\partial p_{i j}}\right)(I-P+\Pi)^{-1} \\
& =Z\left(\frac{\partial P}{\partial p_{i j}}-\Pi H^{i j}\right) Z
\end{aligned}
$$

From (13) we have

$$
\frac{\partial \Phi}{\partial p_{i j}}=\boldsymbol{f}\left(\frac{\partial \Pi_{d g}}{\partial p_{i j}}(2 Z-I-\Pi)+\Pi_{d g}\left(2 \frac{\partial Z}{\partial p_{i j}}-\frac{\partial \Pi}{\partial p_{i j}}\right)\right) \boldsymbol{f}^{\top}
$$

Inserting in relation (45) the derivatives which are computed from (43) and (44), we get the desired result. 
Remark. If for fixed $i, j \in E$ we denote $G_{d g}^{i j}=\operatorname{diag}\left\{\partial g_{u} / \partial p_{i j} ; 1 \leq u \leq s\right\}$, where $\partial g_{u} / \partial p_{i j}$ are given by relation (19), then we have $\left(\Pi H^{i j}\right)_{d g}=G_{d g}^{i j}$.

\section{Examples and numerical applications}

Example 1. Consider the state space $E=\{1,2\}$. Let $p_{12}:=p, p_{21}:=q$. Then, $\mathrm{P}$ is irreducible iff $p>0$ and $q>0$. In this case we can easily compute $\sigma^{2}$ using Proposition 2 to conclude that

$$
\sigma^{2}=\frac{p q(2-p-q)}{(p+q)^{3}}(f(1)-f(2))^{2}
$$

Now, if $0<p<1,0<q<1$ and $f(1) \neq f(2)$, then $\sigma^{2}>0$ and, from Theorem

2 , we get the variance in the asymptotic normal distribution of $\sqrt{m}\left(\widehat{\sigma^{2}}(m)-\sigma^{2}\right)$ in the form

$$
\Phi^{\prime} \Gamma^{*}\left(\Phi^{\prime}\right)^{\top}=\frac{p q}{(p+q)^{7}}[u(p, q)+u(q, p)](f(1)-f(2))^{4},
$$

where, $u(p, q)=(1-p)[(q-p)(2-p-q)-2 p]^{2}$.

Example 2. We will illustrate the above achievements by using some numerical examples. For this purpose let

- $E=\{1,2,3,4,5\}$.

- $P=\left(\begin{array}{ccccc}0.6 & 0.3 & 0 & 0.1 & 0 \\ 0.3 & 0.5 & 0.2 & 0 & 0 \\ 0.4 & 0 & 0.4 & 0 & 0.2 \\ 0 & 0.3 & 0 & 0.5 & 0.2 \\ 0 & 0 & 0.2 & 0.2 & 0.6\end{array}\right)$. 


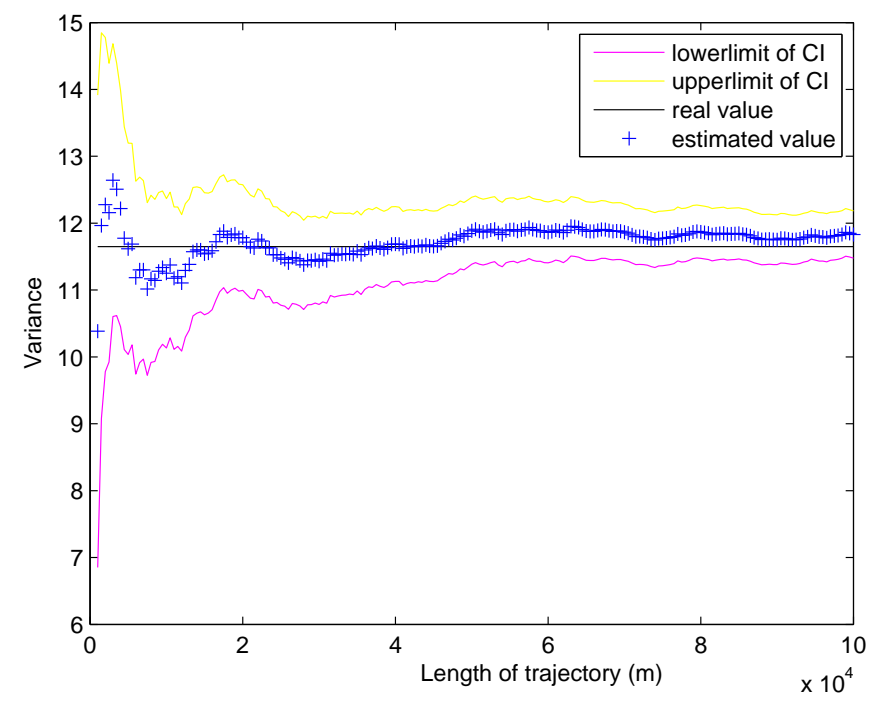

Fig. 1. Variance estimation (95\% CI)

- $f(x)=x$.

The true value for the variance is : $\sigma^{2}=11.6562$

Based on a simulated path of length $10^{5}$ of the above ergodic MC and after recording the successive values of the proposed variance estimator we have made a graphical representation to give a hint for this convergence.

By applying Theorem 2 , we have $\sqrt{m}\left(\widehat{\sigma^{2}}(m)-\sigma^{2}\right) \stackrel{d}{\rightarrow} N(0,3249)$, where we use the integer part of the variance of the limiting distribution.

This helps us in obtaining the $95 \%$ asymptotic confidence intervals by using the fact that $\mathbb{P}\left(\sigma^{2} \in\left[\widehat{\sigma^{2}}(m)-1.96 \sqrt{3249 / m}, \widehat{\sigma^{2}}(m)+1.96 \sqrt{3249 / m}\right]\right)=0.95$ In Figure 1, the confidence intervals are represented as the intervals between the lowest and the highest curve.

Example 3. We consider the random walk in one dimension restricted to a finite set $E=\{0,1, \ldots, s-1\}$. This random walk (or birth and death process in discrete time) is a Markov chain and the transition matrix is of the following 
form

$$
P=\left(\begin{array}{cccccccc}
r_{0} & p_{0} & 0 & 0 & \cdots & 0 & 0 & 0 \\
q_{1} & r_{1} & p_{1} & 0 & \cdots & 0 & 0 & 0 \\
0 & q_{2} & r_{2} & p_{2} & \cdots & 0 & 0 & 0 \\
\vdots & \vdots & \vdots & \vdots & \ddots & \vdots & \vdots & \vdots \\
0 & 0 & 0 & 0 & \cdots & q_{s-2} & r_{s-2} & p_{s-2} \\
0 & 0 & 0 & 0 & \cdots & 0 & q_{s-1} & r_{s-1}
\end{array}\right) .
$$

This Markov chain is irreducible iff $p_{i}, q_{i}>0$, for $1 \leq i \leq s-2$, and $p_{0}, q_{s-1}>0$. If additionally transitions to the same state are not allowed, then $r_{i}=0$ for $0 \leq i \leq s-1$, and the resulting chain is periodic. The Ehrenfest model (see Karlin and Taylor (1975)) can also be considered as a special case of this model. Depending on the kind of model that we study we can adjust a suitable parametric space. We estimate the variance in the CLT for the class of Markov chains described by $P$ with unknown parameters all the non zero off-diagonal elements. As a special case we took $s=30$, reward function $f(x)=x$, and as a true value $P_{0}$ for $P$ we made a random selection of the true values $p_{i}^{0}, q_{i}^{0}$ of the parameters $p_{i}$ and $q_{i}$, with the constraint $0.05 \leq p_{i}^{0}, q_{i}^{0} \leq 0.95$. The true value for the variance is : $\sigma^{2}=2420$.

By applying Theorem 2, we have $\sqrt{m}\left(\widehat{\sigma^{2}}(m)-\sigma^{2}\right) \stackrel{d}{\rightarrow} N\left(0,32027 \times 10^{5}\right)$. In Figure 2 we represent an estimation procedure based on a simulated path of length $10^{5}$, giving also the $95 \%$ confidence intervals. 


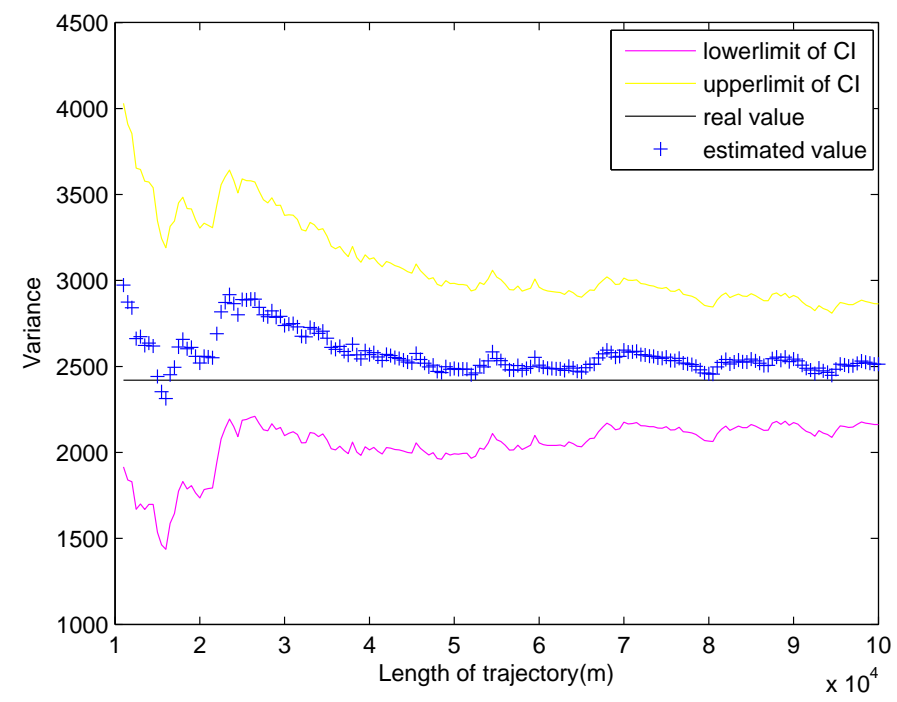

Fig. 2. Variance estimation (95\% CI)

\section{Acknowledgments}

The authors would like to thank the referee and the editor for their helpful comments and suggestions.

\section{References}

Billingsley, P., 1961a. Statistical Inference for Markov Processes. The University of Chicago Press.

Billingsley, P., 1961b. Statistical Methods in Markov Chains. Ann.Math.Stat. $32,12-40$.

Chauveau, D., Diebolt, J., 2003. Estimation of the asymptotic variance in the CLT for Markov Chains. Stochastic Models 19 (4), 449 - 465.

Dacunha-Castelle, D., Duflo, M., 1983. Probabilités et statistiques 2. Masson. Doob, J. I., 1953. Stochastic Processes. Wiley, New York.

Girardin, V., Limnios, N., 2001. Probabilités en Vue des Applications. Vuibert, 
Paris.

Karlin, S., Taylor, H. M., 1975. A First Course in Stochastic Processes. Academic Press, Inc.

Kemeny, J. G., Snell, J., 1976. Finite Markov Chains. Springer-Verlag.

Meyn, S., Tweedy, R., 1993. Markov Chains and Stochastic Stability. SpringerVerlag, London.

Peskun, P. H., 1973. Optimum Monte Carlo sampling using Markov Chains. Biometrika 60, 607-612.

Port, S. C., 1994. Theoretical probability for applications. Wiley, New York.

Resnick, S. I., 1992. Adventures in Stochastic Processes. Birkhäuser, Boston.

Roberts, G. O., Rosenthal, J. S., 2004. General state space Markov Chains and MCMC algorithms. Probability Surveys 1, 20-71.

Sadek, A., Limnios, N., 2002. Asymptotic properties for maximum likelihood estimators for reliability and failure rates of Markov Chains. Communication in Statistics-Theory and Methods 31 (10), 1837-1861.

Seneta, E., 2006. Non-Negative Matrices and Markov Chains. Springer.

Stefanov, V. T., 1995. Explicit limit results for minimal sufficient statistics and maximum likelihood estimators in some Markov processes: Exponential families approach. The Annals of Statistics 23 (4), 1073-1101.

Van Der Vaart, A. W., 1998. Asymptotic Statistics. Cambridge University Press, New York. 